\title{
Stages of vine pruning for vine production of bottle gourd varieties and lines in summer season
}

\author{
M. R. Ali ${ }^{\mathrm{a}}$, G. M. A. Halim ${ }^{\mathrm{b}}$ and H. Mehraj \\ alericulture Division, HRC, BARI, Joydevpur, Gazipur, Dhaka, Bangladesh \\ Olericulture Division, HRC, BARI, Gazipur, Dhaka, Bangladesh \\ ${ }^{c}$ The United Graduate School of Agricultural Sciences, Ehime University, Ehime 790-8556, Japan
}

\ hmehraj02@yahoo.com(Mehraj, H.), Published: 14July 2016

\begin{abstract}
Four bottle gourd varieties and lines ( $V_{1}:$ BARI Lau-3, V V : BARI Lau-4, V $V_{3}:$ LS 0012-5-3 and V4: LS 0026-5-3) and three pruning stages ( $P_{1}$ : Pruning at two vine stage, $P_{2}$ : Pruning at 3 vine stage and $P_{3}:$ Pruning at 4 vine stage) were implied to observe the effect of pruning on the maximization of vine production in bottle gourd. $V_{4}$ produced maximum number of vine (117.2/plant) and highest vine yield (10.2 t/ha) among four varieties while $P_{3}$ produced maximum number of vine (109.2/plant) and highest vine yield (9.1 t/ha) among three pruning techniques. The maximum number of harvested vine (118.0/plant) and highest vine yield (10.73 t/ha) was found from $V_{4} P_{3}$ which was statistically followed by $V_{4} P_{1}$ and the lowest vine yield was recorded from $V_{2} P_{1}(6.62$ t/ha). LS 0026-5-3 along with pruning of terminal shoots four vine stages can be recommended for better vine production in bottle gourd for use as leafy vegetable.
\end{abstract}

Key Words: Lagenaria siceraria, Pruning, Varieties \& lines vine and Yield

Cite article: Ali, M. R., Halim, G. M. A. \& Mehraj, H. (2016). Stages of Vine pruning for vine production of bottle gourd varieties and lines in summer season. Journal of Bioscience and Agriculture Research, 09(01), 792-795.

Article distributed under terms of a Creative Common Attribution 4.0 International License.

\section{Introduction}

Bottle gourd (Lagenaria siceraria) is a commonly cultivated summer seasonal vegetable in Bangladesh belongs to Cucurbitaceae family. Beside fruit production the tender leaves and vines of bottle gourd are also used as a very delicious and highly nutritious vegetable in Bangladesh. Growth and yield performance varied due to the genotypic variation thus facilitate the great opportunity to select the better genotype (Jamal Uddin et al., 2014; Rajesh et al., 1999; Ram et al., 2005) of bottle gourd (Koffi, 2009). Pruning can modify plant growth according to desired levels (Jarrick, 1986). Vine pruning has generally been done for many purposes (Humphries and Vermillion, 1994) more specifically increases the branching of plants. Our current target is to produce more vine of bottle gourd. As bottle gourd tender leaves and vine used as vegetable, so vine pruning may help to increase the production of more leaves and vines by increasing the number of branches. For this circumstance, present study was 
conducted to develop the vine pruning stages in maximizing vine production of bottle gourd for use as leafy vegetable.

\section{Materials and Methods}

The experiment was carried out at the research field of Olericulture Division, Horticulture Research Center, Bangladesh Agricultural Research Institute (BARI), Joydebpur, Gazipur, Dhaka, Bangladesh during summer season of 2014. The experiment consisted two factors, i.e., four bottle gourd varieties and lines ( $\mathrm{V}_{1}$ : BARI Lau-3, $\mathrm{V}_{2}$ : BARI Lau-4, $\mathrm{V}_{3}$ : LS 0012-5-3 and $\mathrm{V}_{4}$ : LS 0026-5-3) and three stages of pruning $\left(\mathrm{P}_{1}\right.$ : Pruning at two vine stage, $\mathrm{P}_{2}$ : Pruning at 3 vine stage and $\mathrm{P}_{3}$ : Pruning at 4 vine stage $)$. Each vine contained main branch during pruning. The seeds of four bottle gourd varieties were sown in poly bags on $10^{\text {th }}$ May 2014 and the seedlings were transplanted in the main field on $5^{\text {th }}$ June 2014 . The experiment was laid out in randomized complete block design with three replications. The unit plot size was $10.0 \times 2.5 \mathrm{~m}$ maintaining $2.0 \times 2.5 \mathrm{~m}$ spacing. The land was fertilized with cow dung, N, P, K, S and Zn @ 20000, 175, 175, 150, 100 and $12 \mathrm{~kg} / \mathrm{ha}$, respectively. The total amount of cow dung, P, S and $\mathrm{Zn}$ and $1 / 3^{\text {rd }}$ of each of $\mathrm{N}$ and $\mathrm{K}$ were applied during final land preparation and in the pit. The rest of $\mathrm{N}$ and $\mathrm{K}$ were applied in four equal installments at 21,35, 55 and 75 days after transplanting. The intercultural operations were done as and when needed. Pruning started after first female flower opening and no fruit was allowed in whole experiment. Data were recorded from three randomly selected plants per treatment per replication on number of vines/plant, weight of vine/plant (kg), vine length $(\mathrm{cm})$, vine diameter $(\mathrm{cm})$, leaf area $\left(\mathrm{cm}^{2}\right)$, internodes length $(\mathrm{cm})$ and vine yield $(\mathrm{t} / \mathrm{ha})$. The collected data on different characters were statistically analyzed using MSTAT-C computer package program and mean differences were determined by Duncan's Multiple Range Test (DMRT) at 5\% level of probability (Gomez and Gomez, 1984).

\section{Results and Discussion}

\section{Main effect of variety on vine production in bottle gourd}

The main effect of variety on vine production in bottle gourd is presented in Table 01. The vine production was statistically influenced by the varieties. Earlier harvest was performed by the variety LS 0012-5-3 (59.00 days).The maximum number of harvested vine (117.2/plant) was produced by LS 0026-5-3 and the highest vine yield was recorded from LS 0026-5-3 (10.2 t/ha). The lowest number of harvested vine (102.8/plant) was observed LS 0012-5-3 and vine yield (7.0 t/ha) was observed in the line BARI Lau-4. The number branches/vines of bottle gourd varied due to the variation of the variety (Singh et al., 2002). Similar results for the variation in vine length were also reported by many researchers in bottle gourd (Munshi and Acharya, 2005; Samadia, 2002; Badade et al., 2001; Mathew et al., 2001; Kumar et al., 2007; Harika et al., 2012), in ash gourd (Sahu et al., 2015), in sponge gourd (Pandey and Singh, 2007), in bitter gourd (Radha Rani, 2014).

\section{Table 01. Varietal effect on the vine production in bottle gourd}

\begin{tabular}{|c|c|c|c|c|c|c|c|c|c|c|c|c|}
\hline \multirow{3}{*}{$\begin{array}{l}\text { Varieties } \\
V_{1}\end{array}$} & \multirow{2}{*}{$\begin{array}{l}\text { Days to } \\
\text { first } \\
\text { harvest }\end{array}$} & \multicolumn{7}{|c|}{ Average vine } & \multirow[b]{2}{*}{$\begin{array}{l}\text { Leaf area } \\
\left(\mathrm{cm}^{2}\right)\end{array}$} & \multirow{2}{*}{\multicolumn{2}{|c|}{$\begin{array}{l}\text { Internodes } \\
\text { length }(\mathrm{cm})\end{array}$}} & \multirow{2}{*}{$\begin{array}{l}\text { Vine } \\
\text { yield } \\
\text { (t/ha) }\end{array}$} \\
\hline & & \multicolumn{2}{|c|}{$\begin{array}{l}\text { number } \\
\text { /plant }\end{array}$} & \multicolumn{2}{|c|}{$\begin{array}{l}\text { weight } \\
\text { (kg/plant) }\end{array}$} & \multicolumn{2}{|c|}{$\begin{array}{l}\text { length } \\
(\mathrm{cm})\end{array}$} & $\begin{array}{l}\text { diameter } \\
(\mathrm{cm})\end{array}$ & & & & \\
\hline & $61.4 \quad \mathrm{a}$ & 111.0 & $\mathrm{~b}$ & 6.4 & $\mathrm{~b}$ & 95.2 & $a b$ & $0.81 \mathrm{ab}$ & 530.7 a & 14.3 & $\mathrm{~b}$ & $9.4 \mathrm{~b}$ \\
\hline $\mathrm{V}_{2}$ & 58.4 & 104.9 & c & 4.7 & d & 96.4 & $a b$ & 0.71 & 391.7 & 13.6 & c & 7.0 \\
\hline $\mathrm{V}_{3}$ & 59.0 & 102.8 & c & 5.2 & c & 93.6 & $\mathrm{~b}$ & 0.81 & 499.6 & 13.9 & c & 7.9 \\
\hline $\mathrm{V}_{4}$ & 59.7 & 117.2 & $\mathrm{a}$ & 6.9 & $\mathrm{a}$ & 97.6 & $d$ & 0.85 & $545.7 \quad \mathrm{a}$ & 14.7 & $\mathrm{a}$ & $10.2 \mathrm{a}$ \\
\hline & 1.1 & 2.6 & & 0.4 & & 3.3 & & 0.13 & 21.2 & 0.4 & & 0.6 \\
\hline CV (\%) & 1.13 & 1.4 & & 4.3 & & 2.0 & & 9.67 & 2.54 & 1.5 & & 4.2 \\
\hline
\end{tabular}

Here, $\mathrm{V}_{1}=$ BARI Lau-3, $\mathrm{V}_{2}=$ BARI Lau-4, $\mathrm{V}_{3}=$ LS 12-5-3 andV $\mathrm{V}_{4}=\mathrm{LS} 26-5-3$

\section{Effect of pruning on vine production in bottle gourd}

The main effect of pruning on vine production in bottle gourd is presented in Table 02. The variation was observed among the different pruning in vine production in bottle gourd. The highest number of harvested vine was recorded in $\mathrm{P}_{3}\left(109.2\right.$ /plant) followed by $\mathrm{P}_{2}(109.0 /$ plant $)$ and lowest in $\mathrm{P}_{3}$ (108.8/plant). The internodes length was maximum in $\mathrm{P}_{1}(14.3 \mathrm{~cm})$ and minimum in $\mathrm{P}_{2}(14.0 \mathrm{~cm})$. The highest vine yield was recorded in $\mathrm{P}_{3}(9.1 \mathrm{t} / \mathrm{ha})$ followed by $\mathrm{P}_{2}(8.5 \mathrm{t} / \mathrm{ha})$ and the lowest yield was 
found in $\mathrm{P}_{1}(8.3 \mathrm{t} / \mathrm{ha})$. Our study showed the significantly different results for studied parameters at different stages of vine pruning. Positive and significant correlation was present between leaf area of vine and weight of bunches in vine (Senthilkumar et al., 2015) i.e., pruning increase the leaf area but severity of pruning decrease the leaf area while pruning severity increased internodal distance (Brandon et al., 2012).

Table 02. Effect of pruning technique on the vine production in bottle gourd

\begin{tabular}{|c|c|c|c|c|c|c|c|c|c|c|c|c|c|c|c|}
\hline \multirow[b]{2}{*}{$\begin{array}{l}\text { Pruning } \\
\text { techniques }\end{array}$} & \multirow{2}{*}{$\begin{array}{l}\text { Days to } \\
\text { first } \\
\text { harvest }\end{array}$} & \multicolumn{8}{|c|}{ Average vine } & \multirow{2}{*}{\multicolumn{2}{|c|}{$\begin{array}{l}\text { Leaf area } \\
\left(\mathrm{cm}^{2}\right)\end{array}$}} & \multirow{2}{*}{\multicolumn{2}{|c|}{$\begin{array}{l}\text { Internodes } \\
\text { length } \\
\text { (cm) }\end{array}$}} & \multirow{2}{*}{\multicolumn{2}{|c|}{$\begin{array}{l}\text { Vine } \\
\text { yield } \\
\text { (t/ha) }\end{array}$}} \\
\hline & & \multicolumn{2}{|c|}{$\begin{array}{l}\text { number } \\
\text { /plant }\end{array}$} & \multicolumn{2}{|c|}{$\begin{array}{l}\text { weight } \\
\text { (kg/plant) }\end{array}$} & \multicolumn{2}{|c|}{$\begin{array}{l}\text { length } \\
(\mathrm{cm})\end{array}$} & \multicolumn{2}{|c|}{$\begin{array}{l}\text { diameter } \\
(\mathrm{cm})\end{array}$} & & & & & & \\
\hline $\mathrm{P}_{1}$ & $60.4 \quad \mathrm{a}$ & 108.8 & $\mathrm{a}$ & 5.6 & $\mathrm{~b}$ & 94.1 & $a$ & 0.79 & $a$ & 499.5 & $\mathrm{a}$ & 14.3 & $\mathrm{a}$ & 8.3 & $\mathrm{~b}$ \\
\hline $\mathrm{P}_{2}$ & 59.8 & 109.0 & $\mathrm{a}$ & 5.7 & b & 96.1 & $\mathrm{a}$ & 0.79 & a & & $\mathrm{a}$ & & $\mathrm{a}$ & 8.5 & $a b$ \\
\hline $\mathrm{P}_{3}$ & 58.8 & 109.2 & $\mathrm{a}$ & 6.1 & $\mathrm{a}$ & 96.9 & $\mathrm{a}$ & 0.80 & a & 474.5 & $\mathrm{~b}$ & 14.0 & & 9.1 & $\mathrm{a}$ \\
\hline & 1.1 & 2.6 & & 0.2 & & 3.3 & & 0.13 & & & & 0.6 & & & \\
\hline CV (\%) & 1.1 & 1.4 & & 4.3 & & 2.0 & & 9.67 & & 2.5 & & 1.5 & & 4.2 & \\
\hline
\end{tabular}

Here, $\mathrm{P}_{1}$ : Pruning at two vine stage, $\mathrm{P}_{2}$ : Pruning at three vine stage, $\mathrm{P}_{3}$ : Pruning at four vine stage

\section{Combined effect of variety and pruning on vine production in bottle gourd}

Significant variations were found in the combined effect of variety and pruning in the vine production in bottle gourd (Table 03). The maximum number of harvested vine per plant (118) was resulted in LS 0026-5-3 along with four vine pruning stage $\left(\mathrm{V}_{4} \mathrm{P}_{3}\right)$. The highest vine yield $(10.7 \mathrm{t} / \mathrm{ha})$ was recorded from the line LS 0026-5-3 when the vines were pruned at four side vine stage $\left(\mathrm{V}_{4} \mathrm{P}_{3}\right)$ which was statistically followed by the same line when the vines were pruned two side vine stage $\left(\mathrm{V}_{4} \mathrm{P}_{1}\right)$ and the lowest vine yield was recorded from the BARI Lau-4 (6.6 t/ha) when it was pruned at two side branch stage $\left(\mathrm{V}_{2} \mathrm{P}_{1}\right)$.

Table 03. Effect of variety and pruning technique combination on the vine production in bottle gourd

\begin{tabular}{|c|c|c|c|c|c|c|c|c|c|c|c|c|c|}
\hline \multirow{3}{*}{$\begin{array}{l}\text { Varieties } \\
\mathrm{V}_{1} \mathrm{P}_{1}\end{array}$} & \multirow{2}{*}{$\begin{array}{l}\text { Days to } \\
\text { first } \\
\text { harvest }\end{array}$} & \multicolumn{7}{|c|}{ Average vine } & \multirow[b]{2}{*}{$\begin{array}{l}\text { - Leaf area } \\
\left(\mathrm{cm}^{2}\right)\end{array}$} & \multirow{2}{*}{\multicolumn{2}{|c|}{$\begin{array}{l}\text { Internodes } \\
\text { length } \\
(\mathrm{cm})\end{array}$}} & \multirow{2}{*}{\multicolumn{2}{|c|}{$\begin{array}{l}\text { Vine } \\
\text { yield } \\
\text { (t/ha) }\end{array}$}} \\
\hline & & \multicolumn{2}{|c|}{$\begin{array}{l}\text { number } \\
\text { /plant }\end{array}$} & \multicolumn{2}{|c|}{$\begin{array}{l}\text { weight } \\
\text { (kg/plant) }\end{array}$} & \multicolumn{2}{|c|}{$\begin{array}{l}\text { length } \\
\text { (cm) }\end{array}$} & $\begin{array}{l}\text { diameter } \\
\text { (cm) }\end{array}$ & & & & & \\
\hline & $61.3 \mathrm{ab}$ & 111.0 & $\mathrm{~b}$ & 6.2 & $\mathrm{~cd}$ & 97.2 & bc & 0.82 abc & $551.3 \mathrm{~b}$ & 14.3 & $\mathrm{c}$ & 9.0 & \\
\hline $\mathrm{V}_{1} \mathrm{P}_{2}$ & 62.3 & 111.3 & b & 6.6 & bc & 96.6 & $\mathrm{c}$ & $0.72 \mathrm{~cd}$ & $.4 \mathrm{ab}$ & 14 & bc & 9.8 & \\
\hline $\mathrm{V}_{1} \mathrm{P}_{3}$ & 60.7 & 110.7 & $\mathrm{~b}$ & 6.3 & c & 91.9 & $\mathrm{~d}$ & $0.88 \quad \mathrm{a}$ & $.5 \mathrm{f}$ & 14 & $\mathrm{~cd}$ & 9.4 & $\mathrm{~cd}$ \\
\hline$V_{2} P_{1}$ & 9.0 & 104.0 & $\mathrm{~cd}$ & 4.4 & $\mathrm{f}$ & 91.8 & $\mathrm{~d}$ & 0.74 & $1 \mathrm{~g}$ & 13 & de & 6.6 & $\mathrm{~h}$ \\
\hline$V_{2} P_{2}$ & $\mathrm{~cd}$ & 105.3 & c & 4.6 & $\mathrm{f}$ & 96.0 & c & $0.72 \mathrm{~cd}$ & $7 \mathrm{~h}$ & 13 & $S$ & 6.8 & gh \\
\hline $\mathrm{V}_{2} \mathrm{P}_{3}$ & 7.7 & 105.3 & $\mathrm{c}$ & 5.0 & e & 101.3 & $\mathrm{a}$ & $0.66 \mathrm{~d}$ & $4 \mathrm{~h}$ & 13 & ef & 7.4 & fg \\
\hline $\mathrm{V}_{3} \mathrm{P}_{1}$ & 0.7 & 103.3 & $\mathrm{~cd}$ & 5.0 & e & 91.4 & $\mathrm{~d}$ & 0.8 & $2 \mathrm{c}$ & 14 & $\mathrm{~cd}$ & 7.6 & $\mathrm{f}$ \\
\hline $\mathrm{V}_{3} \mathrm{P}_{2}$ & 8.7 & 102.3 & $\mathrm{~d}$ & 5.0 & e & 95.3 & c & 0.87 & 3.7 ef & 13 & ef & 7.5 & $\mathrm{f}$ \\
\hline $\mathrm{V}_{3} \mathrm{P}_{3}$ & 57.7 & 102.7 & d & 5.8 & $\mathrm{~d}$ & 94.1 & $\mathrm{~cd}$ & 0.78 & $497.0 \mathrm{de}$ & 13 & ef & 8.7 & $\mathrm{f}$ \\
\hline & 60.6 & 116.7 & $\mathrm{a}$ & 6.9 & $a b$ & 96.0 & $\mathrm{c}$ & 0.81 & $516.6 \mathrm{~cd}$ & 15 & $\mathrm{a}$ & 10.1 & $b$ \\
\hline & 9.3 & 117.0 & $\mathrm{a}$ & 6.5 & bc & 96.4 & $\mathrm{c}$ & 0.83 & $3 a$ & 14 & bc & 9.9 & bc \\
\hline $\mathrm{V}_{4} \mathrm{P}_{3}$ & $59.0 \mathrm{c}$ & 118.0 & $\mathrm{a}$ & 7.2 & $\mathrm{a}$ & 100.3 & $a b$ & $0.91 \mathrm{a}$ & $545.2 \mathrm{~b}$ & 14.7 & $a b$ & 10.7 & $\mathrm{a}$ \\
\hline & 1.1 & 2.6 & & 0.4 & & 3.3 & & 0.13 & & 0.4 & & 0.6 & \\
\hline$C V(\%)$ & 1.1 & 1.4 & & 4.3 & & 2.0 & & 9.67 & 2.54 & 1.5 & & 4.2 & \\
\hline
\end{tabular}

Here, $\mathrm{V}_{1}=$ BARI Lau-3, $\mathrm{V}_{2}=$ BARI Lau-4, $\mathrm{V}_{3}=$ LS 12-5-3 andV $4=$ LS 26-5-3;

$\mathrm{P}_{1}$ : Pruning at two vine stage, $\mathrm{P}_{2}$ : Pruning at three vine stage, $\mathrm{P}_{3}$ : Pruning at four vine stage

\section{Conclusion}

LS 0026-5-3 along with pruning of terminal shoots four vine stages can be recommended for better vine production in bottle gourd for use as leafy vegetable. The present investigation is going on also in summer 2014-15. Again the experiment will be repeated with other varieties and lines for detail study in the next year in both the winter and summer seasons. 


\section{Acknowledgement}

Authors are highly grateful to BARI (Bangladesh Agricultural Research Institute) for giving the opportunity to conduct this experiment. we are also thankful to the persons who helped to the entire experimental work.

\section{References}

[1]. Badade, D. S., Warade, S. D. \& Gaikwad, S. K. (2001). Correlation studies in bottle gourd (Lageneria sicereria (Mol.) Stand). J. Maharashtra Agric Univ. 26(1), 20-22.

[2]. Brandon, S., Archbold, D. D. \& Kurtural, S. K. (2012). Effects of balanced pruning severity on Traminette (Vitis spp.) in a warm climate. Am. J. Enol. Vitic. 63(2), 284-290. http://dx.doi.org/10.5344/ajev.2012.11056

[3]. Gomez, K. A. \& Gomez, A. A. (1984). Statistical procedure for agricultural research (2nd Edn.)Intl. Rice Res. Inst, Willey, A Int. Sci. pp. 28-192.

[4]. Harika, M., Gasti, V. D., Shantappa, T., Mulge, R., Shrimol, A. M., Mastiholi, A. B. \&Kulkarni, M. S. (2012). Evaluation of bottle gourd genotypes [Lagenaria siceraria (Mol.) Standl.] for various horticultural characters.Karnataka J. Agric. Sci. 25(2), 241-244.

[5]. Humphries, E. G. \& Vermillion, D. L. (1994). Pickling cucumber vine pruning treatments and their implicaitons for mechnical harveting. V-37 (1) Tans- ASlA.pp. 71-75.

[6]. Jamal Uddin, A. F. M., Tahidul, M. I., Chowdhury, M. S. N., Shiam, I. H. \& Mehraj, H. (2014). Evaluation of bottle gourd (Lagenaria siceraria) to growth and yield.International Journal of Biosciences, 5(12), 7-11. http://dx.doi.org/10.12692/ijb/5.12.7-11

[7]. Jarrick, J. (1986). Training and pruning. Hort. Sci.p.400.

[8]. Koffi, K. K., Anzara, G. K., Malice, M., Dje, Y., Bertin, P., Baudoin, J. \& Zoro Bi, I. A. (2009). Morphological and allozyme variation in a collection of Lagenaria siceraria (Molina) Standl. from Cote d'Ivoire.Biotechnol. Agron. Soc. Environ. 13(2), 257-270.

[9]. Kumar, S., Singh, R. \& Pal, A. K. (2007). Genetic variability, heritability, genetic advance, correlation coefficient and path analysis in bottle gourd. Indian J. Hort. 64, 163-68.

[10]. Mathew, A., Markose, B. L., Rajan, S. \& Devi, S. N. (2001). Genetic divergence in bottle gourd (Lageneria sicereria (Mol.) Standl).Vegetable Science, 28(2), 121-123.

[11]. Munshi, R. \& Acharyya, P. (2005). Varietal evaluation in bottle gourd genotypes. Indian Agric. 49 (3\&4), 213-221.

[12]. Pandey, R. \& Singh, D. K. (2007). Seasonal effect on fruit yield and study of genetic variability on indigenous germplasm lines of sponge gourd (Luffa cylindrica Roem.). Ann. Agric. Res. New Series, 28(2), 184-191.

[13]. Radha Rani, K. (2014). Performance of bitter gourd genotypes for yield and earliness. Annals of Plant and Soil Research, 16(4), 330-333.

[14]. Rajesh, K., Singh, D. K., Ram, H. H. \& Kumar, R. (1999). Manifestation of heterosis in bottle gourd (Lagenaria siceraria (Mol) Stand L.). Annals of Agril. Res. 20(2), 177-179.

[15]. Ram, D., Singh, R. S., Pandey, S. \& Rai, M. (2005). Study of polygenic traits in off season bottle gourd (Lagenaria siceraria (Mol) Stand L.). Veg. Sci. 32(2), 189-191.

[16]. Sahu, P. K., Sharma, D. \& Nair, S. K. (2015). Performance of ash gourd genotypes for earliness and yield under Chhattisgarh Plains, India. Plant Archives, 15(2), 1157-1160.

[17]. Samadia, D. K. (2002). Performance of bottle gourd genotypes under hot arid environment. Indian J. Hort. 59(2), 167-170.

[18]. Senthilkumar, S., Vijayakumar, R. M., Soorianathasundaram, K. \& Devi, D. D. (2015). Effect of Pruning Severity on Vegetative, Physiological, Yield and Quality Attributes in Grape (Vitis Vinifera L.) - A Review. Curr. Agri. Res. 3(1), 42-54. http://dx.doi.org/10.12944/CARJ.3.1.06

[19]. Singh, D. K. \& Kumar, R. (2002). Studies on the genetic variability in bottle gourd (Lageneria sicereria (Mol.) Standl). Progressive Hort. 34(1), 99-101. 